\title{
Stiffness of Parallel Manipulators With Crank-CONNECTING Rod SySTEM
}

\author{
AginaGa, J.; AltuZARra, O.; Iriarte, X. \& Macho, E.
}

Abstract: Parallel manipulators have better structural and dynamic properties with less structural mass than serial ones, which make them useful for machine-tool applications. Some of them include crank-connecting rod systems in their architecture. This work analyses the stiffness of such parallel manipulators, which is configuration dependent. Results show that highest values of stiffness are reached when at least one crank-connecting rod system arrives at an inverse singularity. Taking advantage of this fact, an approach to motion planning is devised which makes use of inverse singularities to obtain stiffer trajectories. Results show a significant improvement in the stiffness along the trajectory.

Key words: parallel manipulators, stiffness, inverse singularities
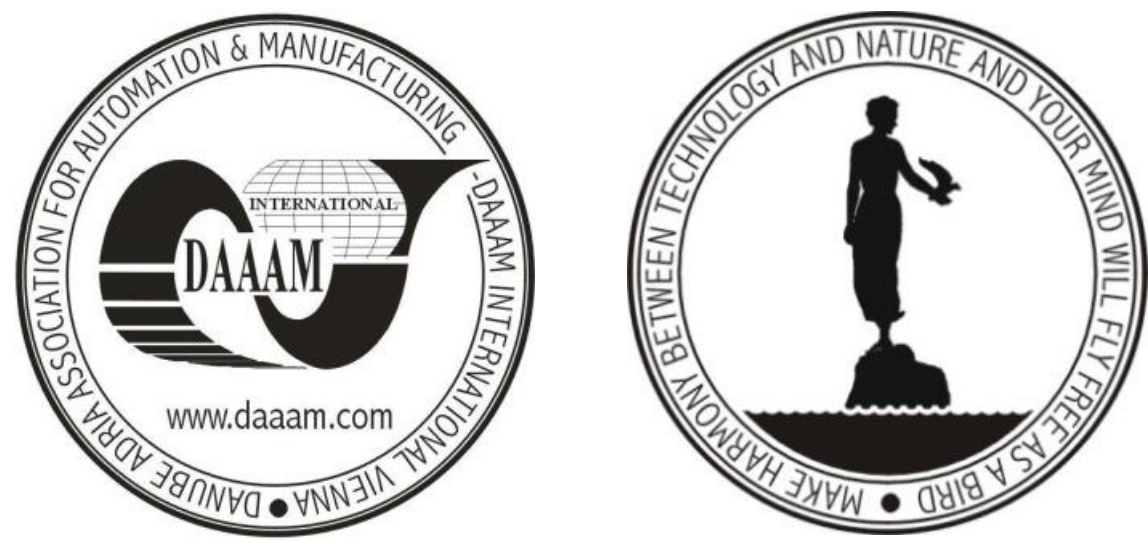

Authors' data: Dipl.-Ing. Aginaga, J[okin]*; Univ.Prof. Altuzarra, O[scar]**; Dr. Iriarte, X[abier]*; Dr. Macho, E[rik]**, *Public University of Navarra, Campus Arrosadia s/n, 31006, Pamplona, Spain, **University of the Basque Country UPV/EHU, Alameda de Urquijo s/n, 48013, Bilbao, Spain. jokin.aginaga@unavarra.es, oscar.altuzarra@ehu.es, xabier.iriarte@unavarra.es, erik.macho@ehu.es

This Publication has to be referred as: Aginaga, J[okin]; Altuzarra, $\mathrm{O}$ [scar]; Iriarte, X[abier] \& Macho, E[rik] (2011). Stiffness of Parallel Manipulators with crankconnecting rod systems, Chapter 38 in DAAAM International Scientific Book 2011, pp. 465-476, B. Katalinic (Ed.), Published by DAAAM International, ISBN 978-3901509-84-1, ISSN 1726-9687, Vienna, Austria

DOI: $10.2507 /$ daaam.scibook.2011.38 


\section{Introduction}

In recent years, many studies have focused on parallel manipulators given their advantages with respect to serial robots. Since their end-effector is sustained by several kinematic chains, they can achieve better structural and dynamic properties with less structural mass. That leads, among other advantages, to higher stiffness, where stiffness can be defined as the capacity of a mechanical system to sustain loads without excessive changes in its geometry. When inputs of a manipulator are locked, the parallel kinematic machine can be considered a structure and its stiffness is then called static stiffness. Since positioning accuracy is strongly dependent on this stiffness, it becomes a key characteristic. Indeed, such characteristic can be essential in certain applications of parallel kinematic machines, such as machine tools, micropositioning devices and mechanisms for surgical procedures.

Several authors have been studying how to quantify the static stiffness of different parallel robots. The way of quantifying the stiffness is usually to build a stiffness matrix which relates an applied external wrench at a reference point with the displacements it produces. One of the first stiffness analysis was carried out by Gosselin (Gosselin, 1990). By means of the Jacobian matrix, stiffness matrix was calculated considering only the stiffness of the actuators, while passive joints and links were assumed to be perfectly rigid. Results demonstrate the dependence of the stiffness on the position in the workspace. In (Di Gregorio \& Parenti-Castelli, 1999), a similar methodology is described, in which links are considered flexible and joints and actuators are taken to be rigid. Later, (Gosselin \& Zhang, 2002) and (Majou et al., 2007) extended these Jacobian-based methods, taking into account stiffness of actuators and links, where link stiffness is simulated by lumped parameters. An analogous methodology was developed in $(\mathrm{Xu} \& \mathrm{Li}, 2008)$ based on screw theory. Ceccarelli \& Carbone (Ceccarelli \& Carbone, 2005) elaborated a similar method constructing a stiffness matrix as the product of several matrices. They used matrices which relate the applied external wrench to local reactions, a second set of matrices which relate these local reactions to local deformation and finally other matrices which relate local deformation to end-effector deformation, and completed it with lumped parameters (Ceccarelli \& Carbone, 2002).

Other authors construct the stiffness matrix by means of a structural or Finite Element formulation. In (Deblaise et al., 2006), structural matrices of the elements are built and assembled including joint stiffness. Finite Element Methods are also used in order to mesh components with a more complex geometry (Huang et al., 2001, Li et al., 2002). Further, Finite Element Methods can be combined with an analytical procedure and experimental results to calculate the static stiffness of a parallel robot (Pinto et al., 2009).

In general, parallel kinematic machines offer higher stiffness with lower moving mass. This work presents a stiffness analysis of parallel mechanisms with crank-connecting rod systems. The fact of having such systems makes these parallel mechanisms have inverse singularities in the workspace. The planar 5R mechanism and the spatial 6-RUS are used as examples in order to show stiffness maps 
calculated by using a Jacobian-based method. Stiffness maps of both 5R and 6-RUS manipulators show that highest values of stiffness occur in poses where crankconnecting rod systems reach inverse singularities. Taking advantage of this fact, an approach to motion planning is devised which makes use of inverse singularities to obtain stiffer trajectories. Two examples are presented in which stiffness is maximised in trajectories with five degrees of freedom (DOF) carried out by the 6RUS manipulator.

\section{Parallel Kinematic Machines with Crank-Connecting Rod System}

The crank-connecting rod system is a simple mechanical device which transforms rotatory motion into a reciprocating one. This system is also used as a subsystem of more complex mechanical devices for several applications. One of these applications are parallel robots, which can include the crank-connecting rod system in a more complex architecture.

A characteristic of the crank-connecting rod system is that it has singularities. These are critical configurations in which the kinetostatic behaviour of a mechanism suddenly changes. Singularities can be divided into two main groups. The first one contains configurations in which the mobile platform loses one or more instantaneous DOFs and, as a consequence, some actuators may have instantaneous velocities that do not produce any motion in the output. The second group corresponds to configurations in which the mobile platform is locally movable even when all the actuated joints are locked. A common nomenclature is to call them inverse singularities and direct singularities respectively. This nomenclature will be used in what follows.

The crank-connecting rod system has two inherent inverse singularities: when the crank and the rod are aligned and when they are superimposed. Unlike direct singularities, inverse singularities are not problematic because the motion of the mechanism remains fully controllable. Moreover, in these positions, the pose of the output link is not affected by a small perturbation in the position of the input joints and it can resist one or more forces or moments without exerting any torque -or forceon the powered joints (Gosselin \& Angeles, 1990). It is also noteworthy that inverse singularities correspond to the limits of the workspace.

A simple planar example of a mechanism including the crank-connecting rod system is the $5 \mathrm{R}$ planar parallel manipulator, commonly known as the five-bar mechanism. It has DOFs defined by five links joined using revolute pairs. Two revolute pairs are actuated and the other three are passive. This mechanism can be understood as two crank-connecting rod systems linked at a singular point. A schematic picture of this mechanism is shown in fig. 1. It is depicted upside-down, down, that is, its fixed points are always over the reference point. Based on this mechanism, Baradat et al. presented a prototype of a light, fast robot for pick-andplace applications (Baradat et al., 2008), with an added passive device for increasing its stiffness out of working plane. 


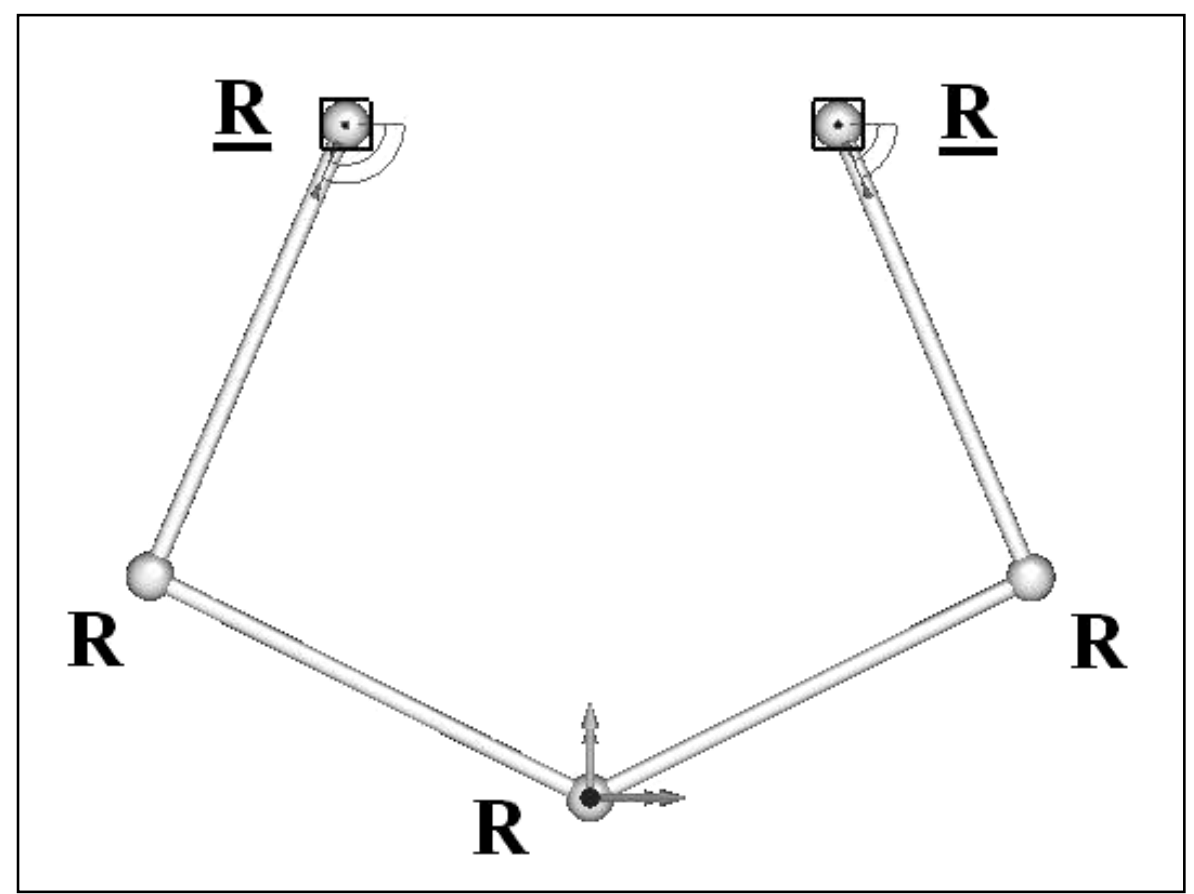

Fig. 1. 5R planar parallel mechanism

Notice that the 5R mechanism reaches an inverse singularity when any of its crank-connecting rod systems is at an inverse singularity. Fig. 2 shows two inverse singularities of the $5 \mathrm{R}$ mechanism. In fig. $2 \mathrm{a}$, only a crank-connecting rod system is at an inverse singularity, whereas in fig. $2 b$ both crank-connecting systems reach an inverse singularity at the same time.

a)

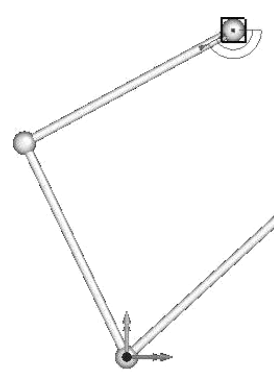

b)

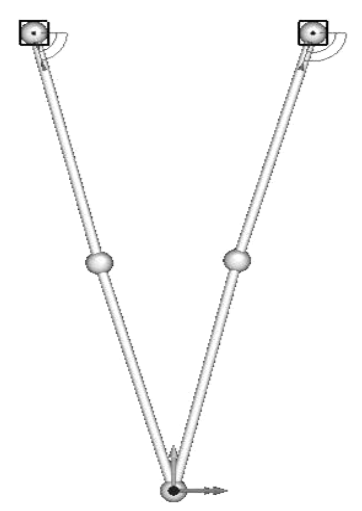

Fig. 2. Inverse singularities of the 5R planar parallel mechanism

A spatial example of parallel manipulator which uses the crank-connecting rod system is the 6-RUS parallel manipulator. It is a mechanism with six DOFs and it was introduced by Hunt (Hunt, 1983). The 6-RUS manipulator is composed of two triangular platforms, one of them fixed to the ground. On the fixed platform there are six rotating actuators $(\underline{\mathrm{R}})$ located on the edges of the triangle. Each actuator is linked to a crank, which is connected to a rod by means of a universal joint (U). The opposite end of the rod is linked to the mobile platform by a spherical joint (S). This architecture can be described as a mobile platform linked to the ground by means of 
six kinematic chains, and these kinematic chains are spatial crank-connecting rod systems. Fig. 3 shows a schematic view of the 6-RUS manipulator.

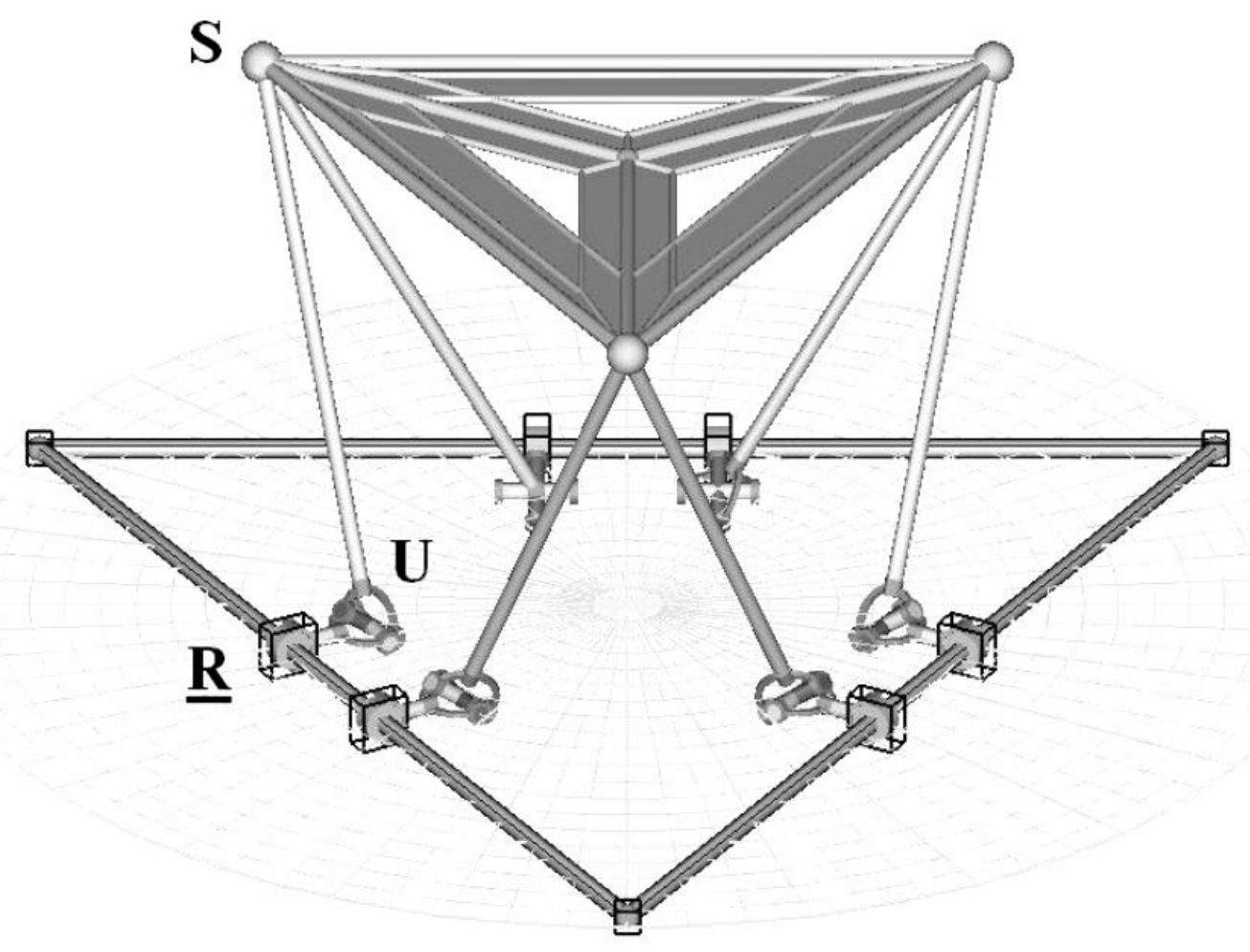

Fig. 3. 6-RUS parallel manipulator

The spatial crank-connecting rod systems of the 6-RUS manipulator cannot reach configurations where the crank and the rod are aligned or superimposed, but this mechanism has inverse singularities in all those configurations in which each rod and its corresponding crank lie in the same plane (Zabalza, 1999). This fact occurs when the crank and the rod are nearly aligned and when they are nearly superimposed, assuming that the rotating actuators are able to perform complete rotations. If the rotating actuators can not accomplish complete rotations, the 6-RUS manipulator may not reach all the inverse singularities.

Since the 6-RUS manipulator has six crank-connecting rod systems, it is possible that some of them arrive at an inverse singularity while the others do not. Zabalza et al. (Zabalza et al., 2003) define a total inverse singularity as all six crankconnecting rod systems reaching an inverse singularity at the same time and a partial inverse singularity as only some of them being at an inverse singularity. Regarding partial inverse singularities, it is noteworthy that the positions of an actuator in which its corresponding crank-connecting rod system is nearly aligned or nearly superimposed depends on the positions of the other actuators, i.e., the rod and the crank can lie together in a range of different planes. Therefore, there is no single value of the position of an actuator for which its corresponding crank-connecting rod system is nearly aligned or for the crank-connecting rod system being nearly superimposed. 
Aginaga, J.; Altuzarra, O.; Iriarte, X. \& Macho, E.: Stiffness of Parallel Manipula...

\section{Stiffness of $5 R$ and 6-RUS manipulators}

The static stiffness matrix $\mathbf{K}$ can be expressed as the relationship between the variation of the pose $\Delta \mathbf{s}$ (three translations and three rotations) of the mobile platform and a wrench $\mathbf{w}$ applied to the mobile platform on the same point.

$$
\mathbf{w}=\mathbf{K} \cdot \Delta \mathbf{s}
$$

Most common way of calculating stiffness is to use this formulation:

$$
\mathbf{K}=\mathbf{J}^{\mathrm{T}} \cdot \mathbf{K}_{\mathbf{l}} \cdot \mathbf{J}
$$

where $\mathbf{J}$ is the Jacobian matrix of the system and $\mathbf{K}_{\mathbf{l}}$ is a matrix with stiffness lumped parameters of the components of the mechanism.

It is commonly known that the stiffness matrix is configuration dependent (Gosselin, 1990), then stiffness matrix must be calculated for every point within the workspace in order to know which configurations are stiffer. Anyway, determining which is the stiffest configuration using the stiffness matrix values is not a simple task, so a scalar performance index is needed. In the literature, scalar parameters of a matrix, such as certain terms of the matrix, the determinant, the norm, the trace or the eigenvalues have been used in order to predict the stiffness by means of a performance index. In this work, the determinant has been considered as the most suitable stiffness performance index, since it involves all the terms of the matrix.

Applying Eq. (2) to the 5R mechanism, its stiffness matrix can be calculated and the determinant can be represented in the $x-y$ plane. In order to adapt the illustration to a logarithmic scale, the logarithm of the determinant is presented in fig. 4.

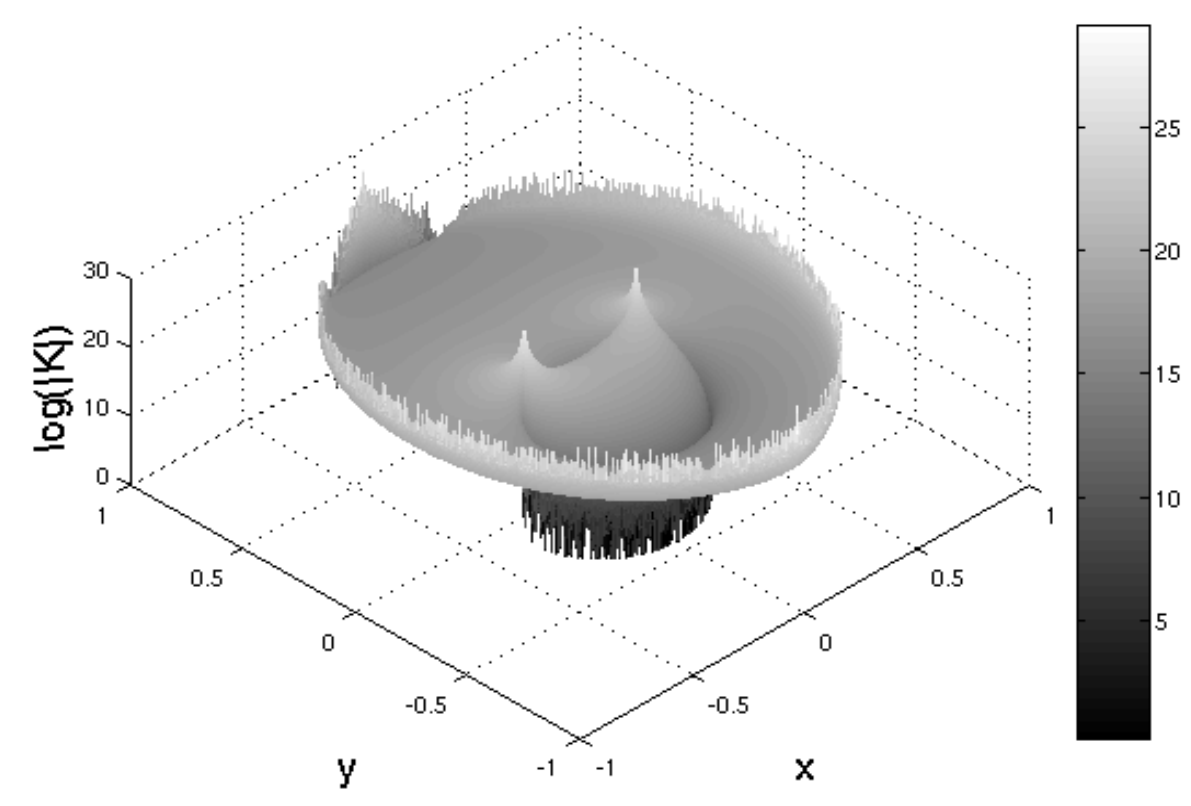

Fig. 4. Stiffness map of the 5R parallel mechanism 
Results show that the highest values of stiffness are reached at the limits of the workspace. In such configurations, at least one limb of the mechanism reaches an inverse singularity. Then, the crank is not under any bending load and the motors are not subjected to any torque, and that is the reason why inverse singularities are stiffer than others. Direct singularities, instead, show a significant loss of stiffness and they must be avoided in order to maintain the robot stability.

The same procedure can be applied to the 6-RUS manipulator. Nevertheless, results of its stiffness matrix are not as simple to depict as those of $5 \mathrm{R}$ mechanism. Since the 6-RUS manipulator has a six-dimensional workspace, it is not possible to depict its overall workspace graphically. Then, it is necessary to fix three DOFs in order to represent the remaining three DOFs. By fixing orientation freedoms, Bonev (Bonev \& Gosselin, 2000) presented an algorithm for the computation of constantorientation workspaces of the 6-RUS manipulator.

Stiffness distributions, instead, must be plotted by fixing four of the six DOFs of the manipulator and depicting remaining two DOFs in horizontal axes with stiffness values on the vertical axis. Fig. 5 shows the logarithms of the determinants of the stiffness matrix for the reachable $\mathrm{x}-\mathrm{y}$ values, for two different values of $\mathrm{z}$ with Euler angles are set to zero.

a)

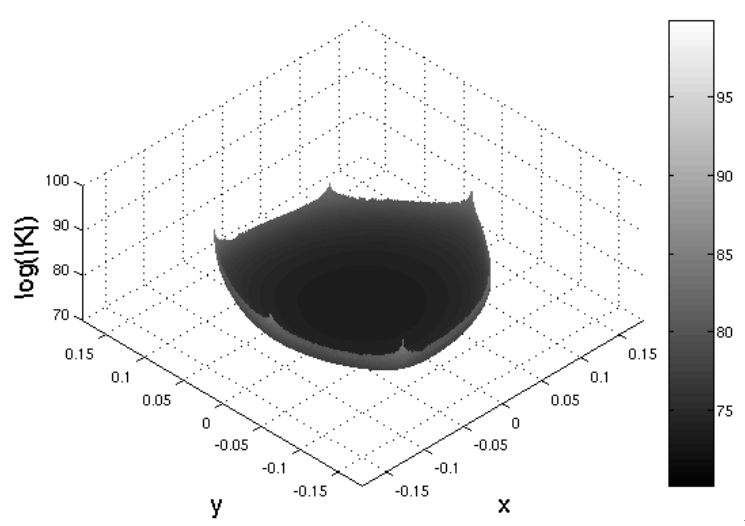

b)

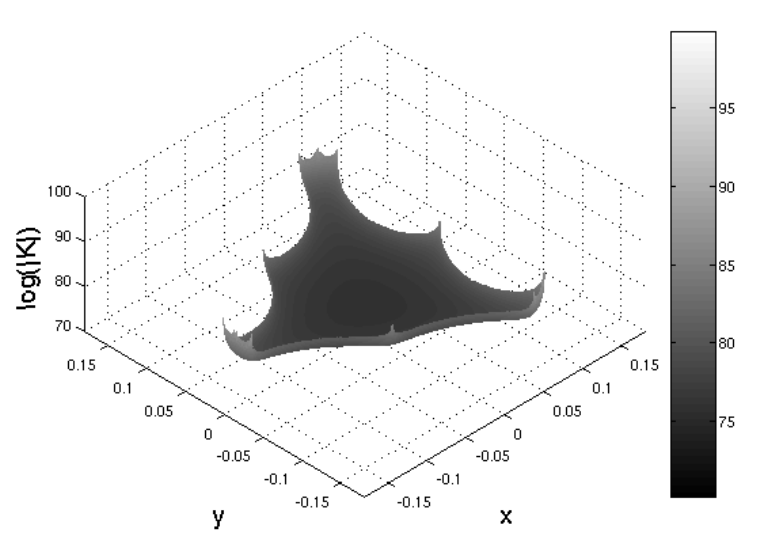

Fig. 5. x-y Stiffness plot of the 6-RUS parallel manipulator

From fig. 5, it can be observed that the distribution of stiffness has $120^{\circ}$ symmetry about the vertical axis. Additionally, the minimum values appear at the central positions while maximum stiffness occurs at the boundary of the workspace. Remember that boundaries correspond to inverse singularities so again inverse singularities reach the highest values of stiffness. Note also that these are partial inverse singularities, thus, not all the crank-connecting rod system reach an inverse singularity. Moreover, the boundaries show also some peaks at certain poses. That means that more than one crank-connecting rod system is at an inverse singularity at those poses.

If $\psi, \theta$ and $\phi$ represent rotations around $\mathrm{x}, \mathrm{y}$ and $\mathrm{z}$ axes respectively, it is also interesting to represent $\phi-z$ and $\theta-x$ stiffness maps. Both are plotted in fig. 6 , the former with $\mathrm{x}, \mathrm{y}, \theta$ and $\psi$ set to zero and the latter with $\mathrm{y}, \mathrm{z}, \phi$ and $\psi$ set also to zero. 
a)

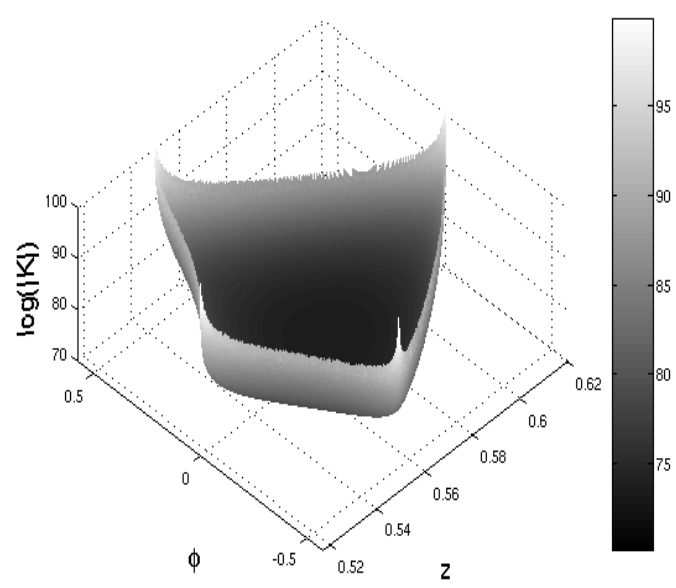

Fig. 6. $\phi$-z and $\theta$-x Stiffness plots of the 6-RUS parallel manipulator

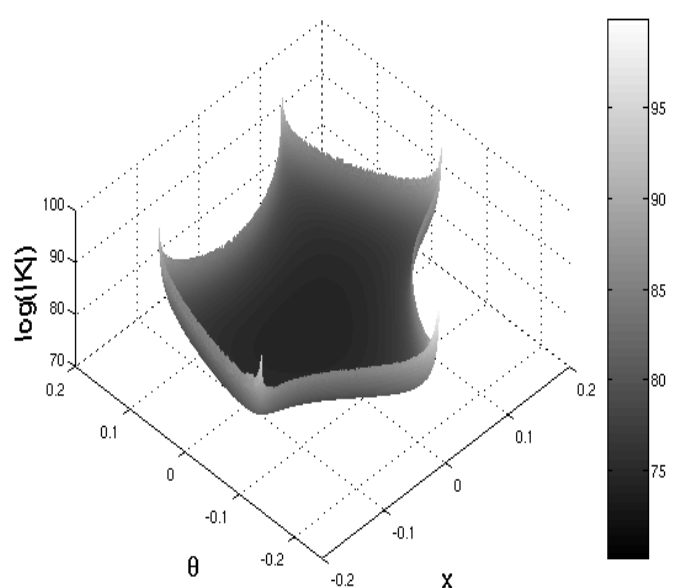

b)

As in Fig. 4, boundaries are also stiffer than central poses. However, maximum values of stiffness are now higher than in the $x-y$ representations. Regarding to fig. $6 \mathrm{a}$, there are four peaks where stiffness values reach very high values. These peaks correspond to four total stationary configurations, where all the crank-connecting rod systems are at an inverse singularity. In the same way, fig. $6 \mathrm{~b}$ shows five peaks of stiffness, one of them higher than the others. This one represents also a total inverse singularity, while the others represent configurations where more than one crankconnecting rod system reaches an inverse singularity. In summary, fig. 6 shows higher values because the manipulator reaches total inverse singularities, which were not reached in the $\mathrm{x}-\mathrm{y}$ representation.

\section{The use of Inverse Singularities in order to improve the Stiffness of a machine}

Both total and partial inverse singularities have been shown to be stiffer than other configurations. Specifically, total inverse singularities reach the highest values of stiffness, but they are located at discrete locations of the workspace. Then, they can only be used as static configurations. Partial inverse singularities, instead, can be maintained while the manipulator moves. An example of this is the $5 \mathrm{R}$ mechanism moving along the limit of its workspace. In such a trajectory, one of its crankconnecting rod systems remains at an inverse singularity. Nevertheless, it is difficult to find out the usefulness of this stiffer trajectory, since it is only applicable to two curves of the workspace.

If the same idea is applied to the 6-RUS manipulator, it is easier to find out its usefulness. If one of the crank-connecting rod systems is constrained to an inverse singularity, the manipulator loses a DOF. Since the 6-RUS manipulator has six DOFs, it is still usable for five, four or three-DOF trajectories. Then, inverse singularities allow us to perform stiffer trajectories of less than six DOFs.

In machine tool applications, it is usual to use five-DOF machines. Since the end-effector of the machine usually has a rotating tool, it is not necessary to include 
Euler angle $\phi$ among the DOFs of the machine. Then, when using the 6-RUS manipulator as a machine tool, said DOF can be used to improve the stiffness.

A way to implement this stiffness-enhancing procedure is to consider the 6RUS manipulator as a positioning table. The part to be machined can be placed on this as shown in fig. 7.

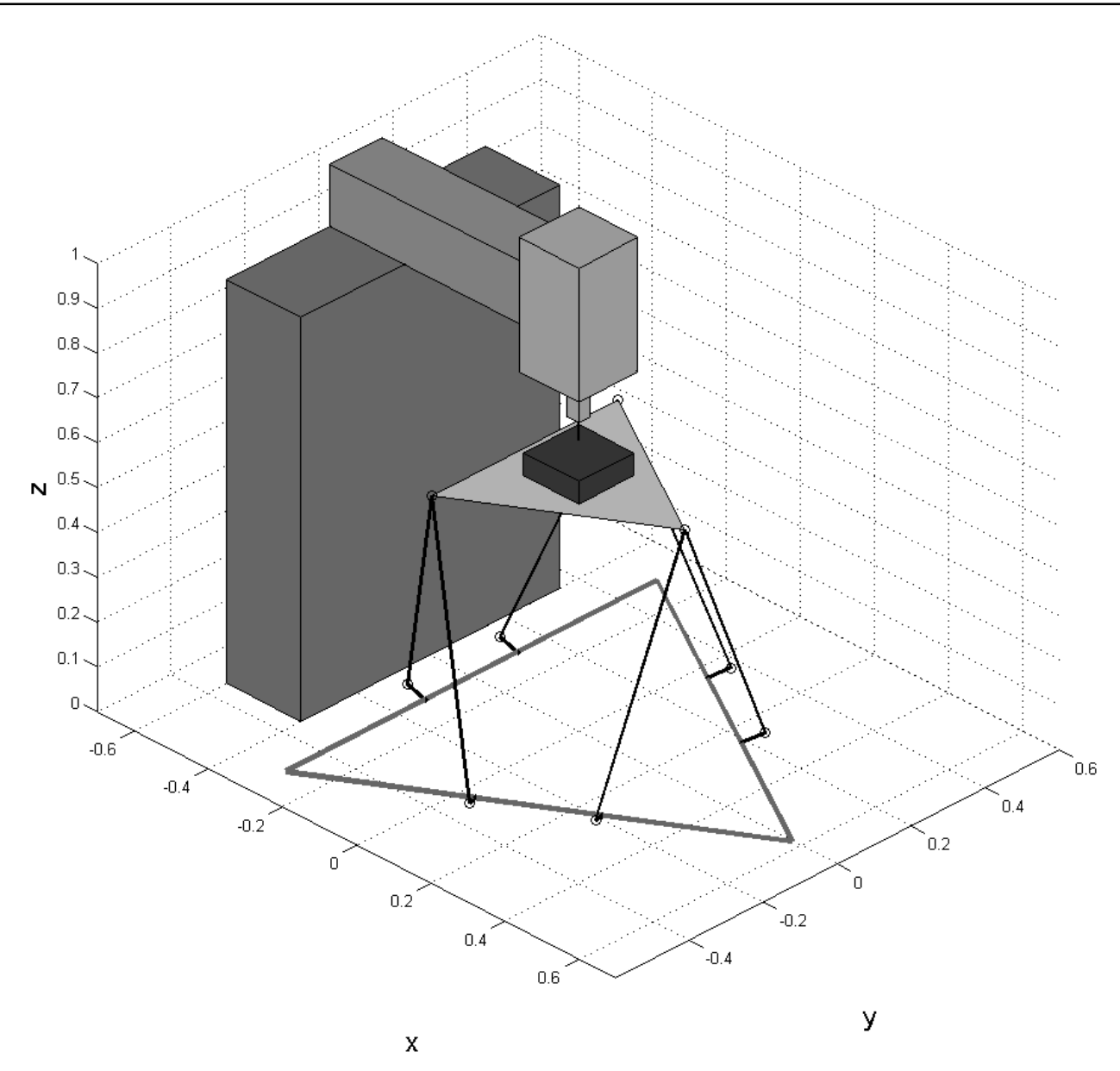

Fig. 7. 6-RUS Manipulator as a positioning table

As a way of example, two five-DOF trajectories of the centre of the mobile platform are analysed: a circular path with Euler angles equal to zero and a conical path in which the vector perpendicular to the mobile platform is taken as the generatrix of a cone. In such trajectories, the value of the Euler angle $\phi$ does not modify the desired trajectory. Therefore, at each discrete position of the trajectory, angle $\phi$ can be used in order to improve the stiffness of the manipulator, that is, in order to reach a partial inverse singularity.

In fig. 8 all possible values of stiffness along circular and conical paths are represented. On the $x$ axis, angle $\alpha$ represents the course of the path; the values of the workspace interval of $\phi$ are plotted on the y axis and the determinant of the stiffness matrix is represented on the $\mathrm{z}$ axis on a $\log$ scale.

As expected, fig. 8 shows that maximum stiffness values are reached at the limits of moving interval of $\phi$. Therefore, in order to reach the stiffest trajectory the Euler angle $\phi$ must be moved to one of its limits at each discrete position. 
a)
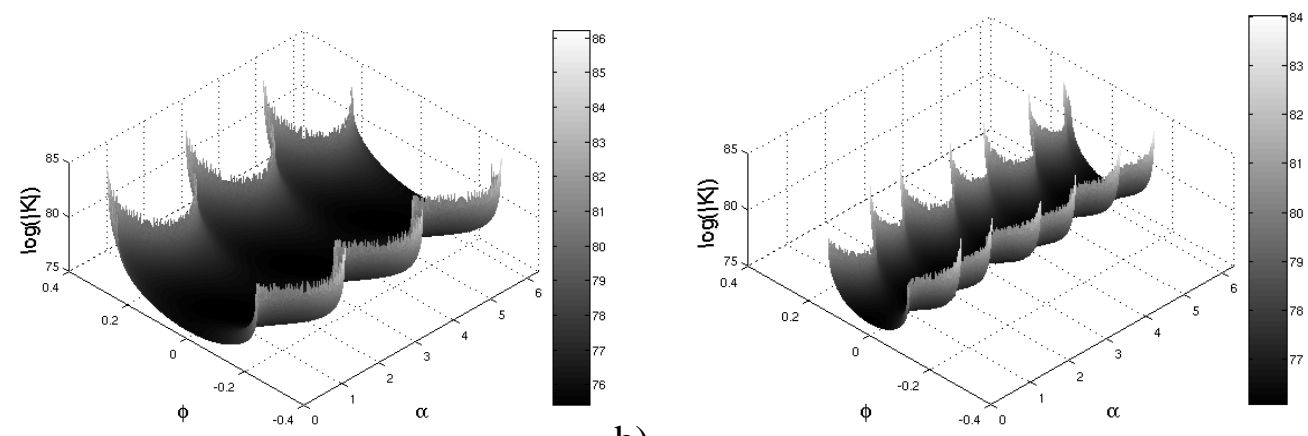

Fig. 8. Reachable stiffness values for circular and conical paths

In fig. 9a stiffness comparison of the circular and conical paths is presented. The solid line shows a reference stiffness value maintaining $\phi=0$; dotted and dashed lines show, respectively, stiffness values along the path with maximum and minimum values of $\phi$, i.e, both limits of its moving interval. When taking the angle $\phi$ to one of the limits of its moving interval, a crank-connecting rod system reaches an inverse singularity. This crank-connecting rod system maintains its inverse singularity until another system reaches an inverse singularity and so on. Stiffness peaks of fig. 9 show instants where two crank-connecting rod systems reach an inverse singularity at the same time.

a)

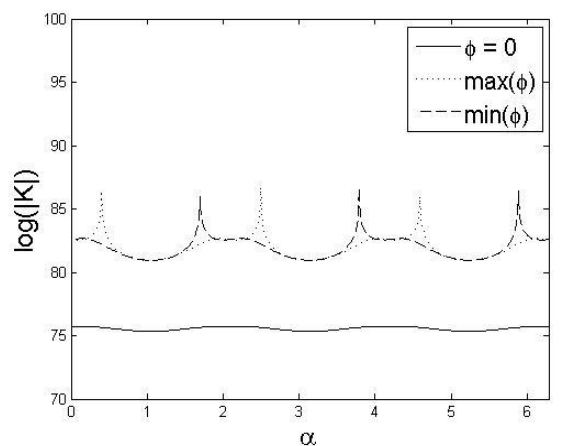

b)

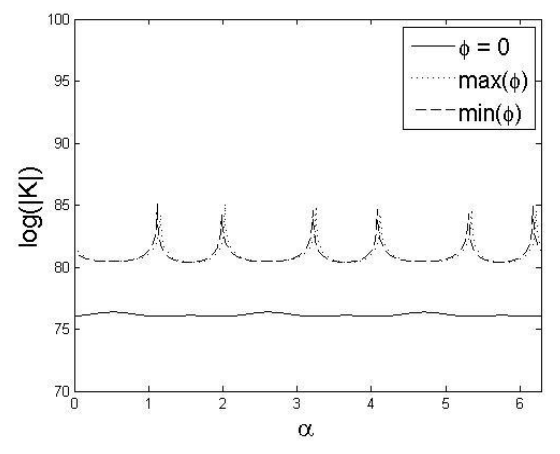

Fig. 9. Stiffness comparison along circular and conical paths

\section{Conclusion}

This works presents a stiffness analysis of parallel mechanism with crankconnecting rod systems. Two examples are chosen, the 5R planar mechanism and the spatial 6- RUS manipulator. Stiffness maps of these mechanisms are calculated and results show that inverse singularities are the stiffest poses of the workspace. Since at these configurations at least one crank-connecting rod system is aligned or nearly aligned, the bending load supported by its crank and the torque supported by its motor are null.

The fact of having greater stiffness at inverse singularities can be used in order to design stiffer five-DOF trajectories for the 6-RUS manipulator. These trajectories are designed by choosing five output freedoms and moving the remaining one to a position where at least one crank-connecting rod system reaches an inverse singularity. Such trajectories can be applied to the 6-RUS manipulator by using it as a 
positioning table of parts to be mechanised. Two trajectories are selected and results show that significant increases in stiffness can be obtained by means of inverse singularities.

The stiffness performance index used is the determinant of stiffness matrix, which has a vague physical meaning. Future work on this field includes the use of a more suitable performance index like, for example, the stiffness in the tool advance direction.

\section{Acknowledgements}

The authors wish to acknowledge the financial support received from the Spanish Government via the Ministerio de Ciencia e Innovacion (Project DPI-200800159), the ERDF of the European Union and the University of the Basque Country (Project GIC07/78).

\section{References}

Baradat, C.; Nabat, V.; Company, O.; Krut, S. \& Pierrot, F. (2008). Par2: a Spatial Mechanism for Fast Planar, 2-dof, Pick-and-Place Applications, In Proceedings of the Second International Workshop on Fundamental Issues and Future Research Directions for Parallel Mechanisms and Manipulators, Montpellier, France, September 2008

Bonev, I. \& Gosselin, C. (2000). A geometric algorithm for the computation of the constant-orientation workspace of 6-RUS parallel manipulators, Proceedings of the ASME Design Engineering Technical Conferences, DETC'2000, Baltimore, MD, USA, September 2000

Ceccarelli, M. \& Carbone, G. (2002). A stiffness analysis for CaPaMan (Cassino Parallel Manipulator). Mechanism and Machine Theory, Vol. 37, No. 5, May 2002, 427-439

Ceccarelli, M. \& Carbone, G. (2005). Numerical and experimental analysis of the stiffness performances of parallel manipulators. In 2nd International Colloquium Collaborative Research Centre 562, Braunschweig, Germany, May 2005

Deblaise, D.; Hernot, X. \& Maurine, P. (2006). A systematic analytical method of PKM stiffness matrix calculation, Proceedings of the 2006 IEEE International Conference on Robotics and Automation, Orlando, USA, May 2006

Di Gregorio, R. \& Parenti-Castelli, V. (1999). Influence of leg flexibility on the kinetostatic behaviour of a 3-DOF fully-Parallel Manipulator, Proceedings of the Tenth World Congress on the Theory of Machines and Mechanisms, IFTOMM'1999, Oulu, Finland, June 1999

Gosselin, C. (1990). Stiffness mapping for Parallel Manipulators. IEEE Transactions on Robotics and Automation, Vol. 6, No 3, June 1990, 377-382

Gosselin, C.M. \& Angeles, J. (1990). Singularity analysis of closed-loop kinematic chains. IEEE Transactions on Robotics and Automation, Vol. 6, No 3, June 1990, 281-290 
Aginaga, J.; Altuzarra, O.; Iriarte, X. \& Macho, E.: Stiffness of Parallel Manipula...

Gosselin, C. \& Zhang, D. (2002). Stiffness analysis of Parallel Mechanisms using a lumped model. International Journal of Robotics and Automation, Vol. 17, No. 1, January 2002, 17-27

Huang, T.; Mei, J.P.; Zhao, X.Y.; Zhou, L.H.; Zhang, D.W. \& Zeng, Z.P. (2001). Stiffness Estimation of a Tripod-based Parallel Kinematic Machine, Proceedings of the 2001 IEEE International Conference on Robotics and Automation, Seoul, Korea. May 2001

Hunt, K.H. (1983). Structural kinematics of in-parallel-actuated robot-arms. Journal of Mechanisms, Transmissions and Automation in Design, Vol. 105, No. 4, December 1983, 705-712

Li, Y-W.; Wang, J-S. \& Wang, L-P. (2002). Stiffness analysis of a Stewart platformbased parallel kinematic machine, Proceedings of the 2002 IEEE International Conference on Robotics and Automation, Washington, DC, USA, May 2002.

Majou, F.; Gosselin, C.; Wenger P. \& Chablat, D. (2007). Parametric stiffness analysis of the Orthoglide. Mechanism and Machine Theory, Vol. 42, No. 3, March 2007, 296-311

Pinto, Ch; Corral, J.; Altuzarra, O. \& Hernandez, A. (2010). A methodology for static stiffness mapping in lower mobility parallel manipulators with decoupled motions. Robotica, 28, 719-735, 2010

$\mathrm{Xu}, \mathrm{Q} \& \mathrm{Li}, \mathrm{X}$. (2008). An investigation on mobility and stiffness of a 3-DOF translational parallel manipulator via screw theory. Robotics and ComputerIntegrated Manufacturing, Vol. 24, No. 3, June 2008, 402-414

Zabalza, I; Pintor, J.M.; Ros, J. \& Jimenez, J.M. (1999), Evaluation of the 64 "Insensitivity" positions for a 6-RKS Hunt-type parallel manipulator, Proceedings of the Tenth World Congress on the Theory of Machines and Mechanisms, IFTOMM'1999, Oulu, Finland, June 1999

Zabalza, I.; Pintor, J.M.; Gil, J.J.; Ros, J. \& Jimenez, J.M. (2003). Total and partial stationary configurations for a 6-RUS Hunt-type parallel manipulator, Proceedings of the Tenth World Congress on the Theory of Machines and Mechanisms, IFTOMM'2003, Tianjin, China, August 2003 